\title{
Aproveitamento de peles de tilápia-do-nilo congeladas e salgadas para extração de gelatina em processo batelada
}

\author{
Adriana Cristina Bordignon ${ }^{1}$, Maria Luiza Rodrigues de Souza Franco², Eliane Gasparino², Edson \\ Minoru Yajima ${ }^{3}$, Ana Paula Del Vesco ${ }^{1}$, Jesui Vergilio Visentainer ${ }^{4}$, Jane Martha Graton Mikcha ${ }^{5}$ \\ 1 Programa de Pós-Graduação em Zootecnia, Universidade Estadual de Maringá, Maringá-PR, 87020-900. \\ 2 Departamento de Zootecnia, Universidade Estadual de Maringá, Maringá-PR, 87020-900. \\ ${ }^{3}$ Gelita do Brasil Ltda. Rod. Maringá Iguaraçu, Km 09 - BR 317. Maringá-PR, 87001-970. \\ ${ }^{4}$ Departamento de Química, Universidade Estadual de Maringá, Maringá-PR, 87020-900. \\ 5 Departamento de Análises Clínicas e Biomedicina, Universidade Estadual de Maringá, Maringá-PR, 87020-900.
}

RESUMO - Objetivou-se caracterizar peles de tilápia-do-nilo, conservadas por congelamento e salga a seco, visando à extração de gelatina em processo batelada. Após a filetagem, as peles foram descarnadas e distribuídas em dois lotes. Um dos lotes foi congelado a $-18{ }^{\circ} \mathrm{C}$ por sete dias e o outro foi salgado e mantido a $25{ }^{\circ} \mathrm{C}$ por sete dias. As peles foram lavadas, pesadas e pré-tratadas em solução de $\mathrm{H}_{2} \mathrm{SO}_{4}$ a10N (pH 3,0), na proporção de 1:6 (pele:água) por uma hora a $24{ }^{\circ} \mathrm{C}$. Extraiu-se a gelatina em banho-maria a $50{ }^{\circ} \mathrm{C}$ por uma hora e retirou-se uma amostra para análise do perfil molecular. $\mathrm{O}$ restante foi congelado a $-18{ }^{\circ} \mathrm{C}$. Foram realizadas análises físico-químicas das peles e das gelatinas líquidas, do perfil molecular com as gelatinas e análises microbiológicas das peles. As peles congeladas e salgadas apresentaram, respectivamente, 78,13 e 76,46\% de umidade; 18,16 e $19,59 \%$ de proteína bruta; 2,26 e 1,90\% de extrato etéreo e 1,44 e 2,06\% de cinzas. Nas gelatinas líquidas extraídas das peles congeladas e salgadas, a umidade foi de 97,68 e 96,08\%, o conteúdo de proteína bruta de 3,18 e 4,12\%, de extrato etéreo 0,29 e $0,18 \%$ e de cinzas de 2,31 e $3,03 \%$, respectivamente. Os valores da força de gel e viscosidade foram maiores para a gelatina de peles salgadas (200 g e 19,02mPas) em comparação à gelatina de peles conservadas pelo congelamento (12,7 g e 9,16mPas). O perfil molecular foi menor na gelatina extraída a partir de peles congeladas, portanto houve perda de $\beta$ e $\gamma$-componentes, que indica grande degradação do colágeno decorrente do método de conservação.

Palavras-chave: composição centesimal, força de gel, gelatina líquida, Oreochromis niloticus, perfil molecular

\section{Utilization of frozen and salted Nile tilapia skin for batch-processed gelatin extraction}

\begin{abstract}
The objective was to characterize Nile tilapia skins, freeze- and dry salt dry-preserved to extract gelatins by batch processing. After filleting, the skins were separated from the meat and distributed into two lots: In one, skins were frozen for 7 days $\left(-18^{\circ} \mathrm{C}\right)$; and in the other, skins were salted for seven days $\left(25^{\circ} \mathrm{C}\right)$. The skins were rinsed, weighed and pretreated in $\mathrm{H}_{2} \mathrm{SO}_{4}$ a10N solution ( $\mathrm{pH} 3.0$ ), at a 1:6 (skin/water) ratio for $1 \mathrm{~h}$ at $24{ }^{\circ} \mathrm{C}$. Gelatin was extracted in water bath at $50{ }^{\circ} \mathrm{C}$ for $1 \mathrm{~h}$, and a sample was removed for molecular profiling; the rest was frozen at $-18{ }^{\circ} \mathrm{C}$. Physical-chemical analyses were carried out on the skins and liquid gelatins, the molecular profile was obtained from the gelatins, and the skins underwent microbiological analyses. Frozen and salted skins showed, respectively: $78.13 \%$ and $76.46 \%$ moisture, $18.16 \%$ and $19.59 \%$ crude protein, $2.26 \%$ and $1.90 \%$ ether extract, and $1.44 \%$ and $2.06 \%$ ash, respectively. For the liquid gelatins extracted from frozen and salted skins, moisture was $97.68 \%$ and $96.08 \%$, crude protein was $3.18 \%$ and $4.12 \%$, ether extract was $0.29 \%$ and $0.18 \%$, and ash was $2.31 \%$ and $3.03 \%$, respectively. Gel strength and viscosity values were higher for salted skins gelatin (200 g and $19.02 \mathrm{mPas}$ ) compared with freeze-preserved skins gelatin (12.7 g and $9.16 \mathrm{mPas})$. The molecular profile was lower in gelatin extracted from frozen skins, which indicates loss of $\beta$ and $\gamma$-components, which indicates considerable collagen decay from that preservation method.
\end{abstract}

Key Words: gel strength, gelatin liquid, molecular profile, Oreochromis niloticus, proximal composition

\section{Introdução}

A tilápia-do-nilo (Oreochromis niloticus) é uma das principais espécies cultivadas no Brasil, devido à sua fácil adaptação ao clima tropical do País. Destaca-se das demais espécies por apresentar carne branca de textura firme, sabor delicado e ausência de odor desagradável (Vannuccini, 1999). O principal produto de sua industrialização é o filé, 
que possui boa aceitação no mercado consumidor por não apresentar espinhos em forma de Y (mioceptos) (Boscolo et al., 2005).

O processo de filetagem gera 60 a $70 \%$ do peso bruto do peixe em resíduos orgânicos de alta qualidade nutricional para a obtenção de diferentes subprodutos, alternativas que geram lucros extras para os produtores e reduzem o efeito prejudicial ao meio ambiente (Vidotti \& Gonçalves, 2006).

A pele de peixe é um subproduto muito utilizado na produção de couro e gelatina. Entretanto, por apresentar grande sensibilidade à degradação, deve ser corretamente conservada para preservar sua qualidade (Giménez et al., 2004). A maioria dos processos de conservação de peles baseia-se na desidratação ou no congelamento, que inibem o desenvolvimento de bactérias e a ação enzimática do processo de autólise (Souza, 2004).

A utilização de gelatinas tem sido difundida ao longo dos anos nas indústrias de alimentos, farmacêuticas, cosméticas e fotográficas. Na indústria alimentícia, tem a função de aumentar a estabilidade, a consistência e a elasticidade dos produtos, devendo apresentar boas propriedades reológicas, como força de gel, viscosidade e ponto de fusão (Choi \& Regenstein, 2000; Gómez-Guillén et al., 2002).

A gelatina de peixe é uma opção para substituição da gelatina tradicional de mamíferos, para uma parcela da população que não se alimenta de produtos derivados de mamíferos e também por questões religiosas e de sanidade. Apresenta algumas características diferenciadas, pois tende a formar géis menos estáveis, em função do conteúdo dos aminoácidos prolina e hidroxiprolina, e propriedades reológicas inferiores às gelatinas de mamíferos, havendo a necessidade de estudos mais aprofundados sobre suas propriedades (Norland, 1990).
Objetivou-se neste estudo aproveitar peles de tilápiado-nilo conservadas por congelamento e salga a seco para realizar a extração de gelatina em processo batelada. Avaliou-se ainda a eficiência dos métodos de conservação por meio da caracterização físico-química, reológica e perfil molecular das gelatinas.

\section{Material e Métodos}

Utilizaram-se aproximadamente $80 \mathrm{~kg}$ de peles brutas de tilápia-do-nilo (Oreochromis niloticus) abatidas com 600 a 800 g de peso, cedidas pelo proprietário do pesqueiro Rancho Verde, situado no Município de Umuarama, Paraná. Após o abate das tilápias, as peles foram retiradas com o auxílio de um alicate e acondicionadas em caixas térmicas com gelo e transportadas até o laboratório de peles e couros localizado na Fazenda Experimental de Iguatemi, pertencente à Universidade Estadual de Maringá. As peles brutas, com músculo, gordura e escamas, foram pesadas e distribuídas em lotes de $3 \mathrm{~kg}$ para cada método de conservação: congelada ou salgada. Após o descarne, realizado com auxílio de colheres para eliminação da camada hipodérmica, os lotes de pele e resíduos sólidos gerados no processo foram novamente pesados. Durante o processo de descarne das peles submetidas ao congelamento à salga a seco, a temperatura da água foi mantida entre 0 e $5^{\circ} \mathrm{C} \mathrm{e} 25^{\circ} \mathrm{C}$, respectivamente.

Após descarne, as peles foram conservadas por congelamento em freezer doméstico $\left(\mathrm{a}-18^{\circ} \mathrm{C}\right)$ e as salgadas a $25{ }^{\circ} \mathrm{C}$ durante sete dias de estocagem. O processo de salga foi realizado em bandejas plásticas perfuradas intercalando camadas de pele e sal. Após a formação de pilhas, adicionou-se uma camada de sal na superfície das peles (Figura 1).
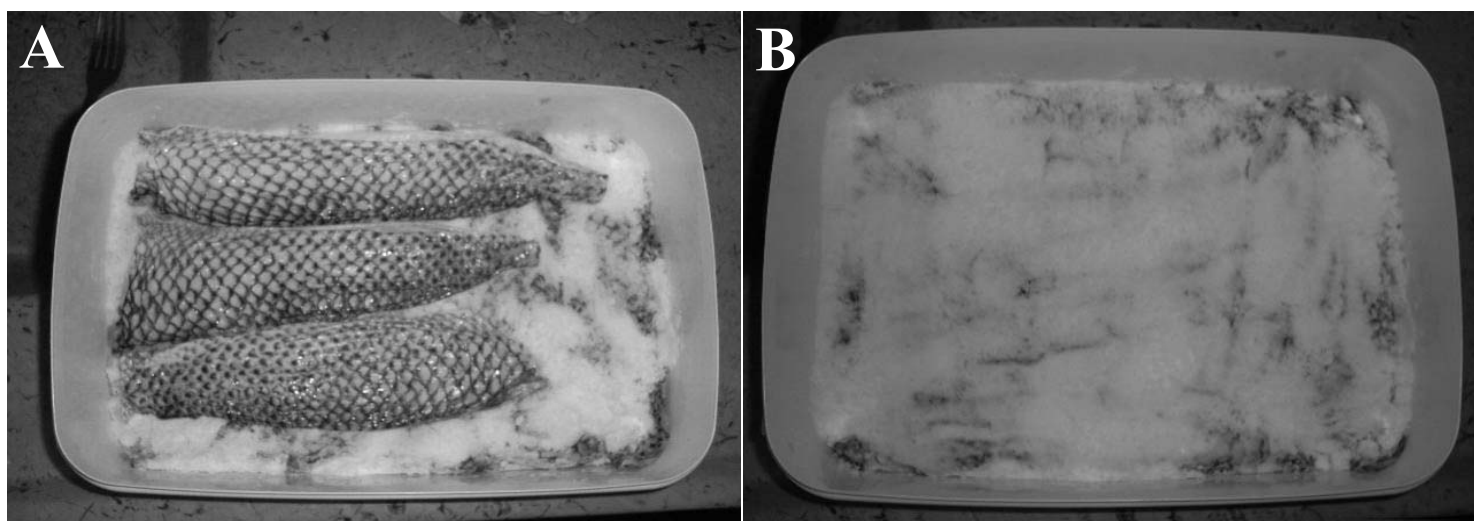

Figura 1 - Processo de salga a seco em peles de tilápia. (A) Peles dispostas em camadas intercaladas de sal; (B) camada de sal sobre as peles.

R. Bras. Zootec., v.41, n.3, p.473-478, 2012 
A extração foi realizada na planta piloto da Empresa Gelita do Brasil, situada no Município de Maringá, Paraná. As peles congeladas foram descongeladas por 12 horas em geladeira e as peles salgadas foram dessalgadas aplicando-se quatro lavagens de 10 minutos. Posteriormente, todas as peles foram lavadas durante 10 minutos, pesadas e imersas em água na proporção de 1 pele: 6 água à temperatura controlada de $24{ }^{\circ} \mathrm{C} \pm 2{ }^{\circ} \mathrm{C}$.

Realizou-se um pré-tratamento ácido por 1 hora, com adição de $\mathrm{H}_{2} \mathrm{SO}_{4} 10 \mathrm{~N}$ até o pH da solução atingir 2,8 a 3,2. Ao término, as peles foram lavadas em água corrente e uma amostra foi retirada para o teste de fervura, que verifica o $\mathrm{pH}$ interno da pele. Após a estabilização do $\mathrm{pH}$, iniciou-se a extração da gelatina em banho-maria, durante uma hora, com temperatura e $\mathrm{pH}$ controlados a $50^{\circ} \mathrm{C} \pm 2^{\circ} \mathrm{C}$ e 4,8 a 5,2, respectivamente. A concentração de sólidos totais foi medida com refratômetro. Após a extração, realizou-se a pesagem da massa de solução de gelatina extraída e da pele não dissolvida. A solução de gelatina foi filtrada a vácuo com filtro de papel e polpa de celulose e dela foi retirada uma amostra para o perfil molecular. Outra amostra foi mantida em freezer a $-18^{\circ} \mathrm{C}$ até o momento da realização das análises e o restante da solução foi concentrado em evaporadora industrial. A solução foi gelificada, triturada e seca em secadora com ar forçado a $25^{\circ} \mathrm{C}$ e depois a $50^{\circ} \mathrm{C}$ por 4 horas, moída e embalada.

Das peles congeladas e salgadas após sete dias de conservação e das gelatinas líquidas congeladas a $-18{ }^{\circ} \mathrm{C}$ foram retiradas amostras para as análises de composição centesimal e mineral.

As análises foram realizadas no Laboratório de Análises de Alimentos e Nutrição Animal - LANA, da Universidade Estadual de Maringá - UEM. Foram realizadas análises dos teores de umidade, cinzas e extrato etéreo, conforme descrito por Cuniff (1998). Os teores de proteína bruta foram avaliados pelo método de Kjeldahl (Silva \& Queiroz, 2002); os de cálcio e ferro foram quantificados conforme descrito por Zhou et al. (1998); e os de fósforo de acordo com Eijsink et al. (1997).

Após filtração da gelatina, retirou-se uma alíquota de $100 \mathrm{~mL}$ e ajustou-se a concentração para $0,1 \mathrm{~mL}$ de gelatina dissolvida em $100 \mathrm{~mL}$ de água deionizada, que foi analisada pela técnica de permeação em gel. O equipamento utilizado foi cromatógrafo para líquido de alta eficiência Waters acoplado a um sistema de deteç̧ão UV-VIS programado com o auxílio do software Millennium 32 para detecção de comprimento de onda de $214 \mathrm{~nm}$. Uma alíquota de $20 \mu \mathrm{L}$ foi injetada com tampão de fosfato (fosfato de sódio/sulfato de sódio) e ajuste de pH com hidróxido de sódio, como fase móvel a um fluxo de $0,6 \mathrm{~mL} /$ minuto, tempo de corrida de 30 minutos a $40^{\circ} \mathrm{C}$ (BSI, 1975).

A força de gel ou bloom é a massa, em gramas, necessária para penetrar em $4 \mathrm{~mm}$ a massa do gel, preparado a uma concentração de $6,67 \%$ de gelatina mantida sob refrigeração a $10^{\circ} \mathrm{C}$, por 17 horas. A solução de gelatina com $6,67 \%$ de sólidos, preparada a partir da gelatina seca em frascos específicos a $60^{\circ} \mathrm{C}$ para completa dissolução e posteriormente resfriado a $10^{\circ} \mathrm{C}$. Para a medida da força de gel foi utilizado o medidor de textura (TA-TX2).

A viscosidade foi realizada com solução de gelatina seca a 6,67\% de sólidos a $60{ }^{\circ} \mathrm{C}$, através do tempo de escoamento de $100 \mathrm{ml}$ da através de uma pipeta padrão.

A análise microbiológica foi realizada no Laboratório de Microbiologia e Microscopia de Alimentos, no Departamento de Análises Clínicas da Universidade Estadual de Maringá - UEM, onde se investigou o número mais provável de coliformes totais a $35^{\circ} \mathrm{C}$ e fecais a $45^{\circ} \mathrm{C}$ (NMP/g) e se realizou a contagem de Estafilococus coagulase positiva (ufc/g) segundo APHA (2001) e pesquisa de Salmonella spp em 25 g, segundo Silva et al. (1997), em peles sujas (com a camada hipodérmica) e após descarne (peles limpas).

O delineamento experimental foi inteiramente casualizado com dois tratamentos: $\mathrm{T} 1$ = gelatina extraída de peles congeladas; e T2 = gelatinas extraída de peles salgadas . Para cada tratamento, utilizaram-se $3 \mathrm{~kg}$ de peles. O perfil molecular foi calculado pela média das amostras e a microbiologia foi realizada para avaliar a qualidade da matéria-prima utilizada para a extração da gelatina. Os resultados da composição centesimal, minerais, força de gel e viscosidade foram submetidos à análise de variância ao nível de 0,05 de probabilidade, utilizando o programa estatístico SAEG versão 8.0.

\section{Resultados e Discussão}

A umidade encontrada nas peles congeladas e salgadas foi de $781,3 \mathrm{~g} / \mathrm{kg}(78,13 \%)$ e 764,6 $\mathrm{g} / \mathrm{kg}(76,46 \%)$, respectivamente, valores superiores aos encontrados por Souza (2004) em peles de tilápia-do-nilo conservadas por congelamento (68,2 a 70,19\%). Muyonga et al. (2004) relataram valores de 72,7 a $68,4 \%$ para jovens e adultos de "nilo perch" (Lates niloticus). Para a corvina e "shortfin scad” (Decapterus macrosoma), os teores de umidade foram, respectivamente, de 62,3 e 60,4\% (Cheow et al., 2007). A umidade elevada ocorreu em virtude dos processos de lavagens das peles adotados desde a chegada ao laboratório até etapas do processo de extração da gelatina. 
A proteína bruta encontrada nas peles da tilápia-donilo foi de 181,6 g/kg (18,16\%) e 195,7 g/kg (19,57\%) para peles conservadas por congelamento e salga a seco, respectivamente. Segundo Songchotikunpan et al. (2008), é com base no teor da proteína bruta da matéria-prima, que é possível estimar qual será a máxima produção de gelatina extraída.

Muyonga et al. (2004) relataram valores de proteína bruta em peles de "nilo perch" jovens e adultos de 20,3 e $21,3 \%$, respectivamente, superiores aos obtidos por Souza (2004), de 27,4\%, para peles de tilápia-do-nilo.

Cheow et al. (2007) encontraram em pele de corvina e "shortfin scad" $24,8 \%$ e $24,1 \%$ de proteína bruta. Os valores de proteína bruta obtidos neste experimento foram inferiores aos relatados na literatura, entretanto essa redução ocorre devido a uma relação inversamente proporcional entre a umidade e a proteína bruta, portanto, quando a umidade for elevada, a proteína bruta será proporcionalmente baixa.

O extrato etéreo variou entre $19,0 \mathrm{~g} / \mathrm{kg}(1,90 \%)$ e $22,6 \mathrm{~g} / \mathrm{kg}(2,26 \%)$ em peles salgadas e congelada, respectivamente. Souza (2004) relatou para a mesma espécie 2,43\% e Songchotikunpan et al. (2008) valor de $1,1 \%$. Baixos teores de gordura são características desta espécie, considerada magra.

O teor de cinzas em peles congeladas foi de $14,4 \mathrm{~g} / \mathrm{kg}$ $(1,44 \%)$ e nas salgadas 20,6 g/kg (2,06\%). Essa variação entre os dois métodos ocorreu pelo fato de o sal não ter sido removido totalmente durante o processo da dessalga. Valor semelhante foi relatado por Souza (2004) e Bueno et al. (2001), para tilápia-do-nilo, de 1,90\%. Songchotikunpan et al. (2008) relataram 2,1\% de cinzas para "nilo perch".

Os teores de cálcio foram mais elevados nas peles congeladas (2,45 mg/100 g) se comparados aos das peles salgadas (1,073 mg/100 g). Os de ferro e fósforo das peles congeladas foram ligeiramente maiores $(0,025 \mathrm{mg} / 100 \mathrm{~g}$ e 0,024 mg/100 mg) que os teores desses minerais nas peles salgadas $(0,019 \mathrm{mg} / 100 \mathrm{~g}$ e $0,019 \mathrm{mg} / 100 \mathrm{~g})$. Portanto, o método de congelamento das peles foi mais eficiente para preservar os minerais em relação à salga.

A composição centesimal das gelatinas líquidas obtidas após a etapa de extração apresentou variação significativa entre os dois métodos, com exceção da proteína bruta
(Tabela 1). Dessa forma, não houve perdas significativas de colágeno ocasionadas pelos métodos de conservação.

As gelatinas líquidas obtidas a partir de peles congeladas apresentaram significativamente maior teor de umidade $(976,8 \mathrm{~g} / \mathrm{kg})$ se comparadas às salgadas $(960,8 \mathrm{~g} / \mathrm{kg})$. Esse teor elevado de umidade entre as duas gelatinas e quando comparadas à gelatina em pó foi ocasionado pelo fato de as gelatinas estarem na forma líquida e não seca. Também ocorreu em função do processo de hidrólise ácida, no qual as peles são hidratadas por moléculas de água que se ligam à estrutura do colágeno.

A gelatina extraída de peles salgadas apresentou Bloom de 200,1 g significativamente superior ao das peles conservadas por congelamento 12,7 g. Esses resultados estão diretamente relacionados às diferenças do peso molecular entre os tratamentos (Tabela 2).

O Bloom varia de acordo com a espécie de peixe. Jamilah \& Harvinder (2002) encontraram 181 g para a tilápia "black" (Oreochromis mossambicus), valor superior ao relatado por Jamilah \& Harvinder (2002) para a tilápia “red” (Oreochromis nilotica) (128 g). Songchotikunpan et al. (2008) encontraram para a tilápia-do-nilo valores de Bloom elevados em comparação aos das demais espécies (328 g). A pele de bacalhau apresentou $70 \mathrm{~g}$ de Bloom e isso pode estar relacionado ao baixo conteúdo de hidroxiprolina de espécies de peixes marinhos em comparação aos tropicais de água doce, portanto está diretamente relacionada à estabilidade do gel.

A viscosidade da gelatina de peles salgadas é mais elevada $(\mathrm{P}>0,05)$ que a das congeladas (Tabela 2). Essa diferença é diretamente proporcional aos valores de força de gel, portanto ocorre com a degradação da cadeia polipeptídica.

Songchotikunpan et al. (2008) encontraram 17,8 mPas de viscosidade para gelatina extraída de peles de tilápia-donilo, valor semelhante ao encontrado para a gelatina extraída das peles salgadas. Jamilah \& Harvinder (2002) encontraram 7,1 mPas e 3,2 mPas de viscosidade para gelatinas de peles de tilápia "black” e tilápia "red”, respectivamente.

Segundo Zhou \& Regenstein (2004), a viscosidade de gelatina extraída de peles de "Alasca pollock" (Theragra chalcogramma) variou entre 1,56 e 6,62 mPas, devido ao método de extração aplicado. De acordo com Sperling (1985),

Tabela 1 - Composição centesimal das gelatinas líquidas após a extração

\begin{tabular}{lcccc}
\hline Peles & Umidade $(\mathrm{g} / \mathrm{kg})$ & Proteína bruta $(\mathrm{g} / \mathrm{kg})$ & Extrato etéreo $(\mathrm{g} / \mathrm{kg})$ & Cinzas $(\mathrm{g} / \mathrm{kg})$ \\
\hline Congeladas & $976,8 \pm 3,0$ & $31,8 \pm 11,4$ & $2,9 \pm 0,4$ & $23,1 \pm 3,0$ \\
Salgadas & $960,8 \pm 17,6$ & $41,2 \pm 16,4$ & $1,8 \pm 1,4$ & $30,3 \pm 3,9$ \\
Valor P & 0,016 & 0,332 & 0,020 & 0,036 \\
CV $(\%)$ & 1,05 & 42,15 & 11,84 & 15,62 \\
\hline
\end{tabular}


a viscosidade de uma solução de gelatina varia de acordo com o peso, tamanho e a distribuição molecular das proteínas. Johnston-Banks (1990) relatou variação na viscosidade de gelatinas comerciais de 2,0 a 7,0 mPas, podendo chegar até 13,0 mPas para as gelatinas com aplicabilidades mais específicas.

O perfil molecular das gelatinas variou entre os tratamentos. Foram registrados menores valores para a gelatina obtida de peles congeladas $(10.682,75 \mathrm{~g} / \mathrm{mol})$ em comparação às peles salgadas $(18.660,80 \mathrm{~g} / \mathrm{mol})$. Isso ocorre por causa das perdas de $\beta$ e $\gamma$-componentes, que indicam grande degradação do colágeno decorrente do método de conservação. Fernández-Diáz et al. (2003), em estudos com peles de “dover sole” (Solea vulgaris) congeladas a $-12^{\circ} \mathrm{C}$ durante 15 dias, relataram que houve grande degradação do colágeno em função do desaparecimento de polímeros com altos pesos moleculares e surgiu uma quantidade elevada de polímeros com pesos moleculares menores, ao passo que, quando as peles foram preservadas na forma fresca e congeladas $\left(-20^{\circ} \mathrm{C}\right)$, não foi verificada perda destes componentes na gelatina.

Muyonga et al. (2004) observaram que, em gelatinas extraídas de peles de "nilo perch" a temperaturas mais elevadas $\left(70^{\circ} \mathrm{C}\right)$, ocorreu a clivagem de cadeias de proteína

Tabela 2 - Dados de força do gel e viscosidade de gelatinas congeladas e salgadas

\begin{tabular}{lcc}
\hline Gelatinas & Força de gel $(\mathrm{g})$ & Viscosidade $(\mathrm{mPas})$ \\
\hline Congeladas & $12,7 \pm 8,70$ & $9,16 \pm 0,85$ \\
Salgadas & $200,1 \pm 31,35$ & $19,0 \pm 2,78$ \\
Valor P & 0,026 & 0,035 \\
CV $(\%)$ & 21,61 & 14,64 \\
\hline
\end{tabular}

durante o processo de extração e, por esse motivo, o peso molecular das frações de peptídeos manteve-se elevado.

A análise microbiológica foi realizada para avaliar a qualidade da matéria-prima utilizada para a produção de gelatina, uma vez que a degradação da pele, por fatores físicos, químicos ou microbiológicos interferem na qualidade da gelatina final.

As peles sujas apresentaram elevada quantidade de coliformes a $35{ }^{\circ} \mathrm{C}$ e $45^{\circ} \mathrm{C}$ e contagem de Estafilococos coagulase positiva. Após o processo de descarne e lavagem das peles, houve redução dos microrganismos presentes, entretanto, quando as peles foram colocadas em sal marinho por sete dias, ocorreu acréscimo de coliformes totais a $35{ }^{\circ} \mathrm{C}$ de $\left(2,4 \times 10^{3}\right)$ e Estafilococus coagulase positiva $\left(1,7 \times 10^{3}\right)$ (Tabela 3$)$. Esse aumento pode estar relacionado ao $\mathrm{pH}$ do meio e à temperatura das peles na bandeja, que proporcionaram um ambiente favorável para o desenvolvimento desses microrganismos.

O número mais provável de coliformes a $35^{\circ} \mathrm{C}$ foi menor que 3 nas peles congeladas após sete dias e $\left(2,4 \times 10^{3}\right)$ nas peles salgadas após sete dias, enquanto o número mais provável de coliformes a $45^{\circ} \mathrm{C}$ observado nos tratamentos foi menor que 3 nas peles congeladas. Em todos os tratamentos, o número mais provável de coliformes a $45^{\circ} \mathrm{C}$ estava de acordo com a legislação vigente para pescados (ANVISA, 2001).

A contagem de Estafilococos coagulase positiva permitida pela legislação brasileira para pescado in natura é até $10^{3} \mathrm{ufc} / \mathrm{g}$. Tanto na peles sujas quanto nas limpas, a contagem permaneceu dentro dos limites para pescado, uma vez que para peles não há legislação vigente. A investigação de Salmonela spp manteve ausente na peles sujas e após os tratamentos.

Tabela 3 - Análises microbiológicas em peles de tilápia-do-nilo

\begin{tabular}{lcccc}
\hline Peles & Coliformes a $35{ }^{\circ} \mathrm{C}^{1}$ & Coliformes a $45{ }^{\circ} \mathrm{C}^{1}$ & Estafilococus coagulase positiva ${ }^{2}$ & Salmonela $^{\mathrm{spp}}$ \\
\hline Suja (com hipoderme) & $2,4 \times 10^{3}$ & 4 & $3,0 \times 10^{3}$ & Ausente \\
Após descarne (7 a $\left.10{ }^{\circ} \mathrm{C}\right)$ & $4,3 \times 10^{1}$ & 9 & $2,5 \times 10^{2}$ & Ausente \\
Congeladas (7 dias) & $<3$ & $<3$ & $6,0 \times 10^{2}$ & Ausente \\
Após descarne em salmoura & $1,1 \times 10^{3}$ & 9 & $1,0 \times 10^{2}$ & Ausente \\
Salgadas (7dias) & $2,4 \times 10^{3}$ & 4 & $1,7 \times 10^{3}$ & Ausente \\
\hline
\end{tabular}

${ }^{1} \mathrm{NMP} / \mathrm{g}$ - número mais provável; ${ }^{2}$ ufc - unidades formadoras de colônia.

\section{Conclusões}

O método de conservação das peles de tilápia-do-nilo por salga a seco proporciona maior eficiência para extração da gelatina em batelada em comparação ao método de conservação por congelamento.

\section{Agradecimentos}

Ministério da Pesca e Aquicultura (MPA), CNPq e Embrapa pelo apoio na realização deste experimento no projeto Aquabrasil. 


\section{Referências}

AMERICAN PUBLIC HEALTH ASSOCIATION - APHA. Compendium of methods for the microbiological examination of foods. 4.ed. Washington: APHA, 2001. 676p.

BOSCOLO, W.R.; HAYASCHI, C.; MEURER, F. et al. Farinha de resíduos da filetagem de tilápia na alimentação de tilápia do Nilo (Oreochromis niloticus) na fase de reversão sexual. Revista Brasileira de Zootecnia, v.34, n.6, p.1807-1812, 2005.

BRITISH STANDARDS INSTITUTION - BSi. Methods for sampling and testing gelatine (physical and chemical methods). London: BS 757: 1975.

BUENO, C.M.; ALVIM, I.D; KOBERSTEIN, T.C.R.D. et al. Produção de gelatina de tilápia e sua utilização para obtenção de micropartículas contendo óleo de salmão. Brazilian Journal of Food Technology, v.14, n.1, p.65-73, 2001.

AGÊNCIA NACIONAL DE VIGILÂNCIA SANITÁRIA - ANVISA. Resolução RDC $n^{\circ} 12$, de 02 de janeiro de 2001. Regulamento técnico sobre padrões microbiológicos para alimentos. Disponível em: <http://www.anvisa.gov.br>. Acesso em: 02 nov 2009.

CHEOW, C.S.; NORIZAH, M.S.; KYAW, Z.Y. et al. Preparation and characterization of gelatins from the skins of sin croaker (Jhonius dussumieri) and shortfin scad (Decapterus macrosoma). Food Chemistry, v.101, n.1, p.386-391, 2007.

CHOI, S.S.; REGENSTEIN, J.M. Physicochemical and sensory characteristics of fish gelatin. Journal of Food Science, v.65, p.194-199, 2000.

CUNNIFF, P.A. Official methods of analysis of AOAC International. 16.ed. Arlington: Association of Official Analytical Chemists International, 1998. 123p.

EIJSINK, L.M.; KROM, M.D.; LANGE, G.J. The use of sequential extraction techniques for sedimentary phosphorus in eastern. Marine Geology, v.139, p.147-155, 1997.

FERNÁNDEZ-DIÁZ, M.D.; MONTERO, P.; GOMÉZ-GUILLÉN, M.C. Effect of freezing fish skins on molecular and rheological properties of extracted gelatin. Food Hydrocolloids, v.17, p.281-286, 2003.

GIMÉNEZ, B. Storage of dried fish skins on quality characteristics of extracted gelatin. Food Hydrocolloids, v.19, p.958-963, 2004.
GÓMEZ-GUILLÉN, M.C.; TURNAY, J.; FERNÁNDEZ-DÍAS, M.D. et al. Structural and physical properties of gelatin extracted from different marine species: a comparative study. Food Hydrocolloids, v.16, n.1, p.25-34, 2002.

JAMILAH, B.; HARVINDER, K.G. Properties of gelatins from skins of fish-black tilapia (Oreochromis mossambicus) and red tilapia (Oreochromis nilotica). Food Chemistry, v.77, p.81-84, 2002.

JOHNSTON-BANKS, F.A. Gelatin. In: HARRIS, P. (Ed.) Food gels. London: Elsevier Applied Science, 1990. p.233-289.

MUYONGA, J.H.; COLE, C.G.B.; DUODU, K.G. Extraction and physicochemical characterisation of Nile perch (Lates niloticus) skin and bone gelatin. Food Hydrocolloids, v.18, p.581-592, 2004.

NORLAND, R.E. Fish gelatin. In: VOIGHT, M.N.; BOTTA, J.K. (Eds.) Advances in fisheries technology and biotechnology for increased profability. Lancaster: Technomic, 1990. p.325-333.

SILVA, D.J.; QUEIROZ, A.C. Análises de alimentos: Métodos químicos e biológicos. 3.ed. Viçosa, MG: UFV, 2002. 235p.

SILVA, N.; JUNQUEIRA, V.C.A.; SILVEIRA, N.F.A. Manual de métodos de análise microbiológica de alimentos. São Paulo: Varela, 1997. 317p.

SONGCHOTIKUNPAN, P.; TATTIYAKUL, J.; SUPAPHOL, P. Extraction and electrospinning of gelatin from fish skin. International Journal of Biological Macromolecules, v.42, p.247-255, 2008.

SOUZA, M.L.R. Tecnologia para processamento das peles de peixe. Maringá: Eduem, 2004. 59p. (Coleção Fundamentum, 11).

SPERLING, L.H. Introduction to physical polymer science. New York: Wiley, 1985. 845p.

VANNUCCINI, S. El enfoque del nuevo mercado de tilapia: en el mundo Occidental. Panorama Acuícola, v.4, n.3, p.22-25, 1999.

VIDOTTI, R.M.; GONÇALVES, G.S. [2006.] Produção e caracterização de silagem, farinha e óleo de tilápia e sua utilização na alimentação animal. Instituto de Pesca. Available at: <htpp://www.pesca.sp.gov.br>. Accesso em: 10 out 2009.

ZHOU, H.Y. Metal composition in sediments and tilapia collected from Island water of Hong Kong. Water Research, v.32, p.3331-3340, 1998.

ZHOU, P.; REGENSTEIN, J.M. Optimization of extraction conditions for pollock skin gelatin. Journal Food Science, v.69, n.5, p.393-398, 2004 